\title{
Full-Sky Maps of the VHF Radio Sky with the Owens Valley Radio Observatory Long Wavelength Array
}

\author{
Michael W. Eastwood and Gregg Hallinan \\ Department of Astronomy, California Institute of Technology, \\ 1200 E California Blvd, Pasadena, CA 91125, USA \\ email: mweastwood@astro.caltech.edu
}

\begin{abstract}
. 21-cm cosmology is a powerful new probe of the intergalactic medium at redshifts $20 \gtrsim z \gtrsim 6$ corresponding to the Cosmic Dawn and Epoch of Reionization. Current observations of the highly-redshifted 21-cm transition are limited by the dynamic range they can achieve against foreground sources of low-frequency $(<200 \mathrm{MHz})$ of radio emission. We used the Owens Valley Radio Observatory Long Wavelength Array (OVRO-LWA) to generate a series of new modern high-fidelity sky maps that capture emission on angular scales ranging from tens of degrees to $\sim 15$ arcmin, and frequencies between 36 and $73 \mathrm{MHz}$. These sky maps were generated from the application of Tikhonov-regularized $m$-mode analysis imaging, which is a new interferometric imaging technique that is uniquely suited for low-frequency, wide-field, drift-scanning interferometers.
\end{abstract}

Keywords. cosmology: early universe, ISM: HII regions, ISM: supernova remnants, techniques: interferometric, radio continuum: general

\section{Introduction}

Following the Epoch of Recombination, the universe plunged into the Dark Ages. During this time the universe is illuminated only by the faint glow of the cosmic background radiation. The Dark Ages ended when star formation began during the Cosmic Dawn. This early star formation bathed the universe in Ly $\alpha$ photons that, after scattering through the Intergalactic Medium (IGM), allow the 21-cm transition of neutral hydrogen to be used as a probe of the local gas temperature, density, and ionization state. The highly redshifted 21 -cm transition $(z \sim 20)$ therefore promises to open a new window into an unobserved era of the universe - the Cosmic Dawn (Pritchard \& Loeb 2012).

Theoretical expectations for the amplitude of the 21-cm transition from the Cosmic Dawn are typically $\sim 10-100 \mathrm{mK}$ (Fialkov et al. 2014). However, the brightness temperature of foreground galactic and extragalactic emission is typically 5 orders of magnitude larger. For instance the brightness temperature at high galactic latitudes has been measured to be $\sim 2000 \times(\nu / 70 \mathrm{MHz})^{-2.6}$ (Mozdzen et al. 2017). The primary challenge of 21-cm cosmology is obtaining the requisite dynamic range against the blinding glow of this foreground emission.

21-cm cosmology is therefore driving a growing demand for high-resolution, highfidelity low-frequency $(\nu<200 \mathrm{MHz})$ sky maps. These sky maps are essential for constructing and testing foreground filtering and removal techniques, as well as in nonredundant gain calibration routines, which can introduce large systematic errors in the event of an incomplete sky model (Barry et al. 2016; Ewall-Wice et al. 2017).

The Owens Valley Radio Observatory Long Wavelength Array (OVRO-LWA) is a 288-element low-frequency (30 to $85 \mathrm{MHz}$ ) interferometer located at the Owens Valley 


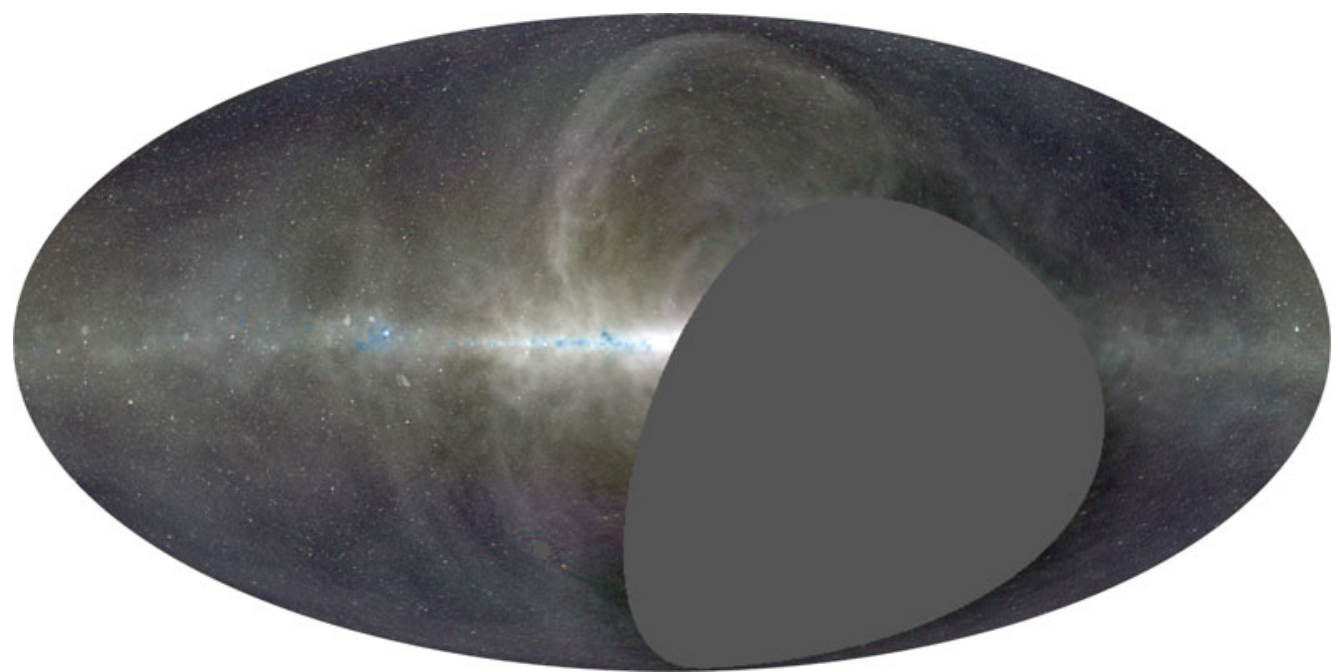

Figure 1. A Mollweide-projected image of the sky created from three of the sky maps generated using the OVRO-LWA and Tikhonov-regularized $m$-mode analysis imaging. This image shows the variety of sources contributing to the 21-cm cosmology foreground: diffuse galactic synchrotron emission, supernova remnants, free-free absorption from HII regions, and thousands of point-like radio galaxies and quasars.

Radio Observatory (OVRO) near Big Pine, California. We used the OVRO-LWA to generate a set of eight new low-frequency sky maps (evenly spaced between $36.528 \mathrm{MHz}$ and $73.152 \mathrm{MHz}$ ) using a new interferometric imaging technique known as Tikonovregularized $m$-mode analysis imaging (Eastwood et al. 2017).

\section{2. m-Mode Analysis Imaging}

Any interferometric imaging algorithm seeks to invert Equation 2.1, which relates the measured correlation $V_{\nu}^{i j}$ between antennas numbered $i$ and $j$ at frequency $\nu$ to the brightness of the sky $I_{\nu}(\hat{r})$ in the direction $\hat{r}$. Generally this is an integral equation depending on the response pattern of the antennas $A_{\nu}(\hat{r})$ and the baseline vector $\vec{b}_{i j}$ that connects the two antennas.

$$
V_{\nu}^{i j}=\int_{\text {sky }} A_{\nu}(\hat{r}) I_{\nu}(\hat{r}) \exp \left(2 \pi i \hat{r} \cdot \vec{b}_{i j} / \lambda\right) \mathrm{d} \Omega,
$$

$m$-mode analysis (Shaw et al. 2014, 2015) exploits a symmetry of drift-scanning interferometers to convert Equation 2.1 from an integral equation into a block-diagonal matrix equation that relates the observations (ie. the $m$-modes) to the spherical harmonic coefficients of the sky brightness (Equation 2.2). The transfer matrix describes the response of the interferometer to the sky including its bandpass, antenna beams, and baseline distribution.

$$
\overbrace{\left(\begin{array}{c}
\vdots \\
m-\text { modes } \\
\vdots
\end{array}\right)}^{v}=\overbrace{\left(\begin{array}{ccc}
\ddots & & \\
& \text { transfer matrix } & \\
& & \ddots
\end{array}\right)}^{B} \overbrace{\left(\begin{array}{c}
\vdots \\
a_{l m} \\
\vdots
\end{array}\right)}^{a}
$$


Table 1. A summary of the generated all-sky maps.

\begin{tabular}{|c|c|c|c|c|c|}
\hline \multirow[b]{2}{*}{ \# } & \multirow{2}{*}{$\stackrel{\nu}{\mathrm{MHz}}$} & \multirow{2}{*}{$\begin{array}{l}\Delta \boldsymbol{\nu}^{1} \\
\mathrm{MHz}\end{array}$} & \multicolumn{2}{|c|}{$\mathbf{F W H M}^{2}$} & \multirow{2}{*}{$\begin{array}{c}\text { Noise }^{3} \\
\text { mJy/beam }\end{array}$} \\
\hline & & & $\delta=0^{\circ}$ & $\delta=+75^{\circ}$ & \\
\hline 1 & 36.528 & 0.024 & $26.0^{\prime} \times 19.1^{\prime}$ & $19.8^{\prime} \times 18.7^{\prime}$ & 799. \\
\hline 2 & 41.760 & 0.024 & $23.3^{\prime} \times 17.5^{\prime}$ & $18.3^{\prime} \times 17.4^{\prime}$ & 824. \\
\hline 3 & 46.992 & 0.024 & $20.9^{\prime} \times 16.3^{\prime}$ & $17.6^{\prime} \times 16.9^{\prime}$ & 717 . \\
\hline 4 & 52.224 & 0.024 & $18.7^{\prime} \times 15.2^{\prime}$ & $16.0^{\prime} \times 15.8^{\prime}$ & 814. \\
\hline 5 & 57.456 & 0.024 & $18.0^{\prime} \times 14.9^{\prime}$ & $15.7^{\prime} \times 15.4^{\prime}$ & 819. \\
\hline 6 & 62.688 & 0.024 & $17.8^{\prime} \times 15.0^{\prime}$ & $15.7^{\prime} \times 15.4^{\prime}$ & 843. \\
\hline 7 & 67.920 & 0.024 & $17.6^{\prime} \times 15.0^{\prime}$ & $15.8^{\prime} \times 15.6^{\prime}$ & 894. \\
\hline 8 & 73.152 & 0.024 & $18.6^{\prime} \times 15.1^{\prime}$ & $16.6^{\prime} \times 16.1^{\prime}$ & 598. \\
\hline
\end{tabular}

Notes:

${ }^{1}$ Bandwidth used to construct the map. As described in the text, each map is constructed from a single frequency channel $(24 \mathrm{kHz})$.

${ }^{2}$ The full-width half-maximum (FWHM) of the synthesized beam at the specified declination (major axis $\times$ minor axis).

${ }^{3}$ Measured with a jackknife and splitting the dataset into even- and odd-numbered integrations. This estimate therefore includes all noise sources that act on the timescale of a single 13 second integration (eg. thermal, ionospheric, etc.).

The block diagonal structure of the transfer matrix means that this equation can be divided into smaller independent equations for a large computational savings. However, Equation 2.2 cannot be inverted without additional regularization. This is because an interferometer located in the northern hemisphere cannot see a patch of sky centered on the southern celestial pole. An additional regularization constraint must therefore be applied to prefer a unique solution for the sky. In this work we applied Tikhonov regularization to force unmeasured parts of the sky to zero (Eastwood et al. 2017).

\section{Sky Maps}

We presented the application of $m$-mode analysis to the OVRO-LWA. Using a 28hour dataset beginning at 2017-02-17 12:00:00 UTC time, we constructed eight maps of the sky at frequencies evenly spaced between $36.528 \mathrm{MHz}$ and $73.152 \mathrm{MHz}$. These maps are a $10 \times$ improvement in angular resolution relative to comparable maps at these frequencies (eg. Dowell et al. 2017), and an example image can be seen in Figure 1. The properties of all eight sky maps are summarized in Table 1. These maps are intended to be useful for calibration and foreground removal in 21-cm cosmology. As such they will be made publicly available on the Legacy Archive for Microwave Background Data Analysis (LAMBDA) †.

The flux scale is tied to an absolute spectrum of Cyg A (Baars et al. 1977), and checked against other low-frequency flux calibrators (Scaife \& Heald 2012; Perley \& Butler 2017). We find that the flux-scale is consistent to within 5-10\%. We compared our maps against existing sky maps at these frequencies (Guzmán et al. 2011; Dowell et al. 2017) and find that the diffuse galactic synchrotron emission is consistent to within $10 \%$. Variable refractive offsets from the ionosphere are responsible for the appearance of $\sim 10 \%$ artifacts within $1^{\circ}$ in the vicinity of bright point sources at $36.528 \mathrm{MHz}$.

Future iterations will incorporate total power radiometry, additional bandwidth and integration time (potentially improving the thermal noise by $10 \times$ ), and improved angular resolution (due to the planned expansion of the OVRO-LWA to $2.5 \mathrm{~km}$ baselines).

$\dagger$ https://lambda.gsfc.nasa.gov/product/foreground/ovrolwa_radio_maps_info.cfm 


\section{References}

Baars, J. W. M., Genzel, R., Pauliny-Toth, I. I. K., \& Witzel, A. 1977, A\&A, 61, 99

Barry, N., Hazelton, B., Sullivan, I., Morales, M. F., \& Pober, J. C. 2016, MNRAS, 461, 3135

Dowell, J., Taylor, G. B., Schinzel, F. K., Kassim, N. E., \& Stovall, K. 2017, MNRAS, 469, 4537

Eastwood, M. W., Anderson, M. M., Monroe, R. M., et al. 2017, ArXiv e-prints, arXiv:1711.00466

Ewall-Wice, A., Dillon, J. S., Liu, A., \& Hewitt, J. 2017, MNRAS, 470, 1849

Fialkov, A., Barkana, R., Pinhas, A., \& Visbal, E. 2014, MNRAS, 437, L36

Guzmán, A. E., May, J., Alvarez, H., \& Maeda, K. 2011, A\&SA, 525, A138

Mozdzen, T. J., Bowman, J. D., Monsalve, R. A., \& Rogers, A. E. E. 2017, MNRAS, 464, 4995 Perley, R. A., \& Butler, B. J. 2017, ApJS, 230, 7

Pritchard, J. R., \& Loeb, A. 2012, Reports on Progress in Physics, 75, 086901

Scaife, A. M. M., \& Heald, G. H. 2012, MNRAS, 423, L30

Shaw, J. R., Sigurdson, K., Pen, U.-L., Stebbins, A., \& Sitwell, M. 2014, ApJ, 781, 57

Shaw, J. R., Sigurdson, K., Sitwell, M., Stebbins, A., \& Pen, U.-L. 2015, Phys. Rev. D, 91, 083514 